CERN-PPE/97-131

9 October 1997

\title{
Measurement of the neutral kaon regeneration amplitude in carbon at momenta below $1 \mathrm{GeV} / c$
}

\author{
CPLEAR Collaboration
}

A. Angelopoulos ${ }^{1)}$, A. Apostolakis ${ }^{1)}$, E. Aslanides ${ }^{11)}$, G. Backenstoss ${ }^{2)}$, P. Bargassa $^{13)}$, O. Behnke ${ }^{17)}$, A. Benelli ${ }^{9)}$, V. Bertin ${ }^{11)}$, F. Blanc ${ }^{7,13)}$, P. Bloch $^{4)}$, P. Carlson $^{15)}$, M. Carroll ${ }^{9)}$, J. Carvalho ${ }^{5)}$, E. Cawley ${ }^{9)}$, S. Charalambous ${ }^{16)}$, G. Chardin ${ }^{14)}$, M.B. Chertok ${ }^{3)}$, A. Cody ${ }^{9}$, M. Danielsson ${ }^{15)}$, M. Dejardin ${ }^{14)}$, J. Derre ${ }^{14)}$, A. Ealet ${ }^{11)}$, C. Eleftheriadis ${ }^{16)}$, I. Evangelou ${ }^{8)}$, L. Faravel $^{7)}$, R. Ferreira-Marques ${ }^{5)}$, W. Fetscher ${ }^{17)}$, M. Fidecaro ${ }^{4)}$, A. Filipčič ${ }^{10)}$, D. Francis ${ }^{3)}$, J. Fry ${ }^{9)}$, E. Gabathuler ${ }^{9)}$, R. Gamet ${ }^{9)}$, D. Garreta ${ }^{14)}$, H.-J. Gerber ${ }^{17)}$, A. Go ${ }^{15)}$, A. Haselden ${ }^{9}$,

P.J. Hayman ${ }^{9)}$, F. Henry-Couannier ${ }^{11)}$, R.W. Hollander ${ }^{6)}$, E. Hubert ${ }^{11)}$, K. Jon-And ${ }^{15)}$, P.-R. Kettle ${ }^{13)}$, P. Kokkas ${ }^{4)}$, R. Kreuger ${ }^{6)}$, R. Le Gac ${ }^{11)}$, F. Leimgruber ${ }^{2)}$, A. Liolios ${ }^{16)}$, E. Machado ${ }^{5)}$, I. Mandić(1) ${ }^{10}$, N. Manthos ${ }^{8)}$, G. Marel ${ }^{14)}$, M. Mikuž ${ }^{10)}$, J. Miller $^{3)}$, F. Montanet ${ }^{11)}$, A. Muller ${ }^{14)}$, T. Nakada ${ }^{13)}$, B. Pagels ${ }^{17)}$, I. Papadopoulos ${ }^{16)}$, P. Pavlopoulos ${ }^{2)}$, J. Pinto da Cunha $^{5)}$, A. Policarpo ${ }^{5)}$, G. Polivka ${ }^{2)}$, R. Rickenbach ${ }^{2)}$, B.L. Roberts ${ }^{3)}$, T. Ruf ${ }^{4)}$, L. Sakeliou ${ }^{1)}$, P. Sanders ${ }^{9)}$, M. Schäfer ${ }^{17)}$, L.A. Schaller ${ }^{7)}$, T. Schietinger ${ }^{2)}$, A. Schopper ${ }^{4)}$,

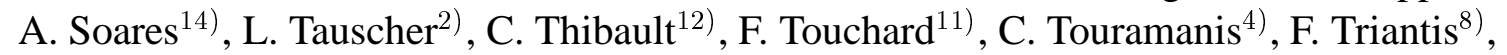
E. Van Beveren ${ }^{5)}$, C.W.E. Van Eijk ${ }^{6)}$, S. Vlachos ${ }^{2)}$, P. Weber ${ }^{17)}$, O. Wigger ${ }^{13)}$, M. Wolter ${ }^{17)}$, C. Yeche ${ }^{14)}$, D. Zavrtanik ${ }^{10)}$ and D. Zimmerman ${ }^{3)}$

\begin{abstract}
The neutral kaon regeneration amplitude in carbon at momenta between 250 and $750 \mathrm{MeV} / \mathrm{c}$ was determined by measuring the interference of inherent and coherently regenerated $\mathrm{K}_{\mathrm{S}}$ amplitudes. This interference appears in the rates of initially pure (tagged) $\mathrm{K}^{0}$ and $\overline{\mathrm{K}}^{0}$ decaying to $\pi^{+} \pi^{-}$after crossing a carbon absorber.
\end{abstract}


Introduction

Regeneration of neutral kaons [1,2] plays an important role in most experiments that measure $\mathrm{CP}$ violation in the $\mathrm{K}$ system, either for the fact that regeneration is employed to produce a $K_{S}$ beam or because $K_{S}$ regenerated in detector material can fake $C P$ violating $K_{L}$ decays. In the case of the CPLEAR experiment the $\mathrm{K}_{\mathrm{S}}$ amplitude that is regenerated in the detector interferes with the inherent one from the produced $\mathrm{K}^{0}\left(\overline{\mathrm{K}}^{0}\right)$ states causing a small but not always negligible change in the decay rates.

The regenerating power of a medium is given by the difference, $\Delta f=f(0)-\bar{f}(0)$, between the nuclear forward scattering amplitudes of $\mathrm{K}^{0}$ and $\overline{\mathrm{K}}^{0}$, which is also called regeneration amplitude [3]. Direct measurements of $\Delta f$ are available for several materials of interest but only for kaon momenta above $2 \mathrm{GeV} / c$ [4], well beyond the CPLEAR momentum range ( $\lesssim 750 \mathrm{MeV} / c$ ). In view of the complete lack of experimental data for the desired momentum range, it was decided to dedicate the last data taking period of the CPLEAR experiment to the measurement of regeneration in carbon. Carbon nuclei account for about two thirds of the regeneration effect in the unmodified detector. Other contributions are mainly due to nitrogen and oxygen which have very similar nuclear properties.

In this letter we report the results of these measurements. An analysis including the application of analyticity and dispersion relations will appear elsewhere.

\section{The experiment}

The CPLEAR experiment is designed to study CP, T and CPT symmetries by measuring time-dependent decay rate asymmetries of $\mathrm{CP}$ and $\mathrm{T}$ conjugate processes [5]. Initially pure $\mathrm{K}^{0}$ and $\overline{\mathrm{K}}^{0}$ states are produced concurrently via the antiproton annihilations $(\mathrm{p} \overline{\mathrm{p}})_{\text {rest }} \rightarrow \mathrm{K}^{0} \mathrm{~K}^{-} \pi^{+}$ and $(\mathrm{p} \overline{\mathrm{p}})_{\text {rest }} \rightarrow \overline{\mathrm{K}}^{0} \mathrm{~K}^{+} \pi^{-}$. The strangeness of the neutral kaon is tagged by the charge sign of the accompanying kaon. The experiment uses an intense $200 \mathrm{MeV} / \mathrm{c}$ antiproton beam $\left(\approx 10^{6} \overline{\mathrm{p}} / \mathrm{s}\right)$ from the Low Energy Antiproton Ring (LEAR) at CERN.

A detailed description of the detector can be found elsewhere [6] and only a brief outline is presented here. The antiprotons are stopped in a cylindrical gaseous hydrogen target (27 bar pressure) which is surrounded by a small proportional chamber. The tracking is performed with two additional layers of proportional chambers, six layers of drift chambers and two layers of streamer tubes. A scintillator-Čerenkov-scintillator sandwich provides input to a fast trigger system to identify the kaon, based on energy loss, time of flight and Čerenkov light measurements. All components are situated inside a solenoid providing a magnetic field of $0.44 \mathrm{~T}$. The

\footnotetext{
1) University of Athens, Greece

2) University of Basle, Switzerland

3) Boston University, USA

4) CERN, Geneva, Switzerland

5) LIP and University of Coimbra, Portugal

6) Delft University of Technology, Netherlands

7) University of Fribourg, Switzerland

8) University of Ioannina, Greece

9) University of Liverpool, UK

10) J. Stefan Inst. and Phys. Dep., University of Ljubljana, Slovenia

11) CPPM, IN2P3-CNRS et Université d'Aix-Marseille II, France

12) CSNSM, IN2P3-CNRS, Orsay, France

13) Paul Scherrer-Institut (PSI), Switzerland

14) CEA, DSM/DAPNIA, CE-Saclay, France

15) Royal Institute of Technology, Stockholm, Sweden

16) University of Thessaloniki, Greece

17) ETH-IPP Zürich, Switzerland
} 
arrangement enables the measurement of neutral kaon decay rates in the region of 0 to $20 \tau_{\mathrm{S}}$, where $\tau_{\mathrm{S}}$ denotes the $\mathrm{K}_{\mathrm{S}}$ mean life. Fast and efficient background rejection is achieved online by the multilevel trigger system.

The carbon absorber (regenerator) has the shape of a segment of a hollow cylinder with an inner radius of $5.8 \mathrm{~cm}$, a thickness of $2.5 \mathrm{~cm}$, a length of $25.5 \mathrm{~cm}$ and an opening angle of $115^{\circ}$. To maximize the regenerating effect within the constraints of the detector design it was positioned close to the inner wall of the first large proportional chamber (PC1). The opening angle was chosen to enable the selection of events with neutral kaons regenerated in the carbon but no charged primary tracks $\left(\mathrm{K}^{ \pm}\right.$and $\left.\pi^{\mp}\right)$ that crossed the absorber. The topology of a typical regeneration event is depicted schematically in Fig. 1. The density of the carbon was $1.85 \mathrm{~g} / \mathrm{cm}^{3}$ and shown to be homogeneous by computerized tomographic imaging.

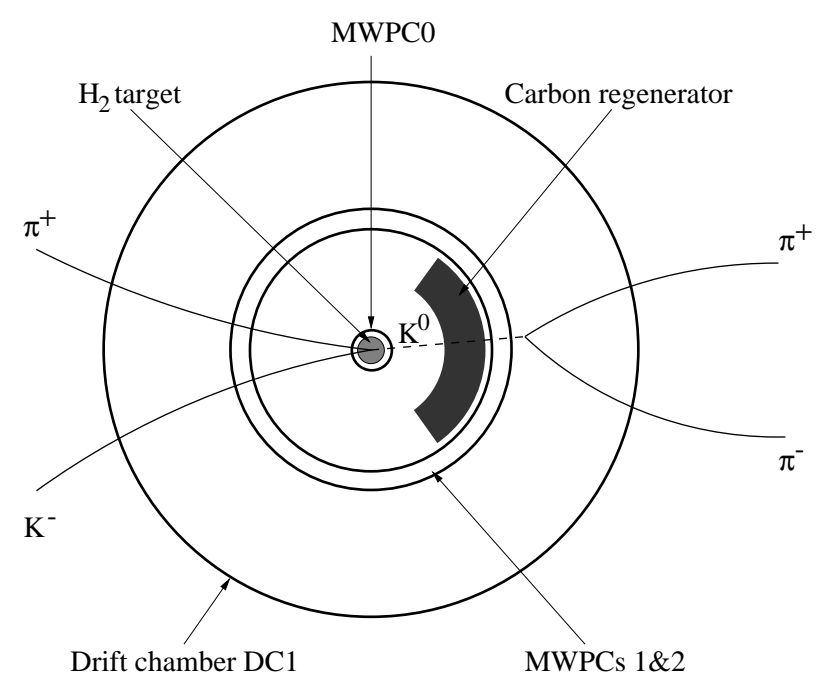

Figure 1: The topology of a typical regeneration event in an expanded view of the inner detector.

The final position and orientation of the absorber inside the detector was measured with a (radial) precision of $0.5 \mathrm{~mm}$ through reconstructed photon conversions and verified to a similar precision by conventional surveying methods.

\section{Overview of the method}

With the unique possibility of tagging the strangeness of neutral kaons at the time of their production, CPLEAR measures asymmetries between the decay rates of initial $\mathrm{K}^{0}$ and $\overline{\mathrm{K}}^{0}$ into various final states as a function of the decay eigentime $\tau$. In this analysis we are concerned with the $\pi^{+} \pi^{-}$final state, i.e., we look at the asymmetry

$$
A_{+-}(\tau)=\frac{\bar{N}_{\pi^{+} \pi^{-}}(\tau)-N_{\pi^{+} \pi^{-}}(\tau)}{\bar{N}_{\pi^{+} \pi^{-}}(\tau)+N_{\pi^{+} \pi^{-}}(\tau)}
$$

where $N_{\pi^{+} \pi^{-}}(\tau)$ and $\bar{N}_{\pi^{+} \pi^{-}}(\tau)$ are the numbers of observed $\mathrm{K}^{0}$ and $\overline{\mathrm{K}}^{0}$ decays to $\pi^{+} \pi^{-}$. The interference of $\mathrm{K}_{\mathrm{L}}$ and $\mathrm{K}_{\mathrm{S}}$ amplitudes leads to the well known oscillatory term in $A_{+-}(\tau)$ [7],

$$
\left|\eta_{+-}\right| \cos \left(\Delta m \tau-\phi_{+-}\right)
$$

which is an unambiguous sign of CP violation. When the asymmetry is measured in a regenerating medium, additional terms of the form

$$
|\varrho| \cos \left(\Delta m \tau-\phi_{+-}+\arg (\varrho)\right)
$$


appear, where $\varrho$ depends on the properties of the medium and is proportional to $\Delta f$ [8]. The result is a pronounced change of the $A_{+-}$oscillation pattern. This can be regarded as an additional interference, between inherent and regenerated $K_{\mathrm{S}}$ amplitudes, or as an amplification of the asymmetry caused by the unequal interaction of $\mathrm{K}^{0}$ and $\overline{\mathrm{K}}^{0}$ in the medium. If the regenerating medium is only present in a limited range of lifetimes (i.e., the neutral kaons of a given momentum cross an absorber of a certain thickness), there will be a sharp step in the asymmetry in that range (cf. [8]). In our experiment, however, this step is smeared out (see Fig. 2) because the effective distance and thickness of the absorber as encountered by the neutral kaon vary from event to event depending on its emission angle relative to the detector axis. Furthermore, the neutral kaons are not mono-energetic but have a finite range of momenta (Fig. 3).

To isolate the interference terms arising from regeneration in our measurement, we compare the regeneration data with a reference data sample which was recorded before the carbon absorber was installed, i.e., we use two sets of data for the determination of the regeneration amplitudes:

1. the actual regeneration data taken with the absorber installed as described in the previous section and

2. a reference set containing data which was obtained without the absorber, i.e., with the original experimental set-up. This set also forms the larger part of the data used for the determination of the CP violation parameter $\eta_{+_{-}}$[9].

The two sets of data were taken under the same detector and trigger conditions, the only difference being the presence of the absorber and they were also subject to exactly the same selection procedure. The reference data set contains more than three times the number of events of the regeneration data set so that the statistical error is almost entirely given by the statistics of the latter.

\section{$4 \quad$ Data selection}

The offline tools used in the initial selection of $\pi^{+} \pi^{-}$decays are identical to the ones applied in the CP violation analysis [7]: The events must have four tracks with zero total charge and a good reconstruction quality is required for each track and vertex. The transverse momentum of the charged kaon has to be greater than $350 \mathrm{MeV} / c$. A kinematical and geometrical fit with nine constraints is imposed on the remaining events. The constraints require conservation of energy and momentum, the missing mass at the annihilation vertex to be equal to the $\mathrm{K}^{0}$ mass, the intersections of two track helices at the annihilation and decay vertices, respectively, and the neutral kaon momentum to be collinear with the line joining the two vertices. Charged tracks crossing the absorber are corrected for energy loss before applying the fit. A cut on the fit probability substantially improves the signal-to-background ratio and the decay time resolution. Only events with neutral kaon lifetimes above $1 \tau_{\mathrm{S}}$ are retained.

In addition to this standard selection of $\mathrm{K}^{0}\left(\overline{\mathrm{K}}^{0}\right) \rightarrow \pi^{+} \pi^{-}$events we demand, in both data sets, that the direction of the neutral kaon is in the absorber sector and reject events in which one or both of the charged primary tracks cross the volume occupied by the absorber. Furthermore we only accept neutral kaons with momenta greater than $250 \mathrm{MeV} / c$ (see next section).

$3 \times 10^{6}$ events of the regeneration data set satisfy all selection criteria. In two thirds of these events, however, the neutral kaon decays before reaching the absorber, thus yielding no information on the regeneration amplitude. About $10^{7}$ events of the reference data set remain after applying identical selection cuts. 


\section{$5 \quad$ Determination of the regeneration amplitudes}

To account for the variation of the regeneration amplitude $\Delta f$ as a function of $\mathrm{K}^{0}\left(\overline{\mathrm{K}^{0}}\right)$ momentum we divide the data of both sets into five momentum intervals of $100 \mathrm{MeV} / c$ width each. The asymmetry (1) is formed from the regeneration data separately for each momentum interval. Figure 2 shows the resulting five asymmetries. The gain in statistical precision at higher momenta is in part compensated by a loss of sensitivity caused by the decreasing evolution time of the neutral kaon before reaching the absorber. Due to the smearing effects mentioned in Sec. 3, it is not possible to extract the desired information by a fit to an analytical function. Instead, we perform a numerical fit, using the reference data set in the following way:

- For each momentum interval we construct the asymmetry (1) as for the regeneration data but now simulate the regeneration effect for a given value of $\Delta f$ by applying a weight to each event. This weight is calculated individually as the ratio of the expected decay rates with and without the absorber, taking into account the momentum and the path of the neutral kaon in the detector. The evaluation of the corrected decay rates is based on the phenomenology of Ref. [8] with the parameters of the neutral kaon system taken from Ref. [10]. Only coherent regeneration is taken into account as the effect of incoherent (diffractive) regeneration is found to be negligible with our method (see following section).

- The simulation of the regeneration effect on the asymmetries is performed for different values of the real and imaginary parts of $\Delta f$, resulting in an array of asymmetries $A_{+-}^{\operatorname{sim}}(\Delta f, \tau)$.

- These regeneration-simulated asymmetries are now compared with the observed asymmetry $A_{+-}^{\text {obs }}(\tau)$ of the regeneration data by evaluating

$$
\chi^{2}(\Delta f)=\sum_{\tau \text { bins }} \frac{\left(A_{+-}^{\mathrm{sim}}(\Delta f, \tau)-A_{+-}^{\mathrm{obs}}(\tau)\right)^{2}}{\sigma_{\mathrm{sim}}^{2}(\tau)+\sigma_{\mathrm{obs}}^{2}(\tau)}
$$

where the $\sigma \mathrm{s}$ are the statistical errors on the respective asymmetries.

- The pair $(\operatorname{Re}(\Delta f), \operatorname{Im}(\Delta f))$ that yields the smallest value for $\chi^{2}$ is the result of our measurement. The fixed- $\chi^{2}$ curves given by $\chi^{2}=\chi_{\min }^{2}+s^{2}$ in the $\Delta f$ complex plane approximate error contours of a Gaussian distribution for $s$ standard deviations, respectively.

For most neutral kaon momenta, the inherent $\mathrm{K}_{\mathrm{S}}$ amplitude by far outweighs the regenerated one at the time of the decay. This means that the spatial vertex distribution of $\pi^{+} \pi^{-}$decays in the regeneration set is well approximated by the data of the reference set and the weights applied to simulate the regeneration effects are close to one. At very low momenta, however, we find that almost all $\mathrm{K}_{\mathrm{S}}$ decay before reaching the absorber and hence our method is not applicable. We therefore restrict the analysis to neutral kaon momenta above $250 \mathrm{MeV} / c$ where the decay rates after the absorber are still dominated by the inherent $\mathrm{K}_{\mathrm{S}}$ amplitude.

The quantities $\langle\Delta f\rangle_{p_{i}}$ determined in this way are weighted averages according to the momentum distribution $\mathcal{N}(p)$ in the respective interval as found at the entrance of the absorber (see Fig. 3):

$$
\langle\Delta f\rangle_{p_{i}}=\frac{\int_{\Delta p_{i}} \mathrm{~d} p \Delta f(p) \mathcal{N}(p)}{\int_{\Delta p_{i}} \mathrm{~d} p \mathcal{N}(p)}
$$

The differences between these spectrum-weighted means and the centre-of-interval values are small compared to our statistical errors. 

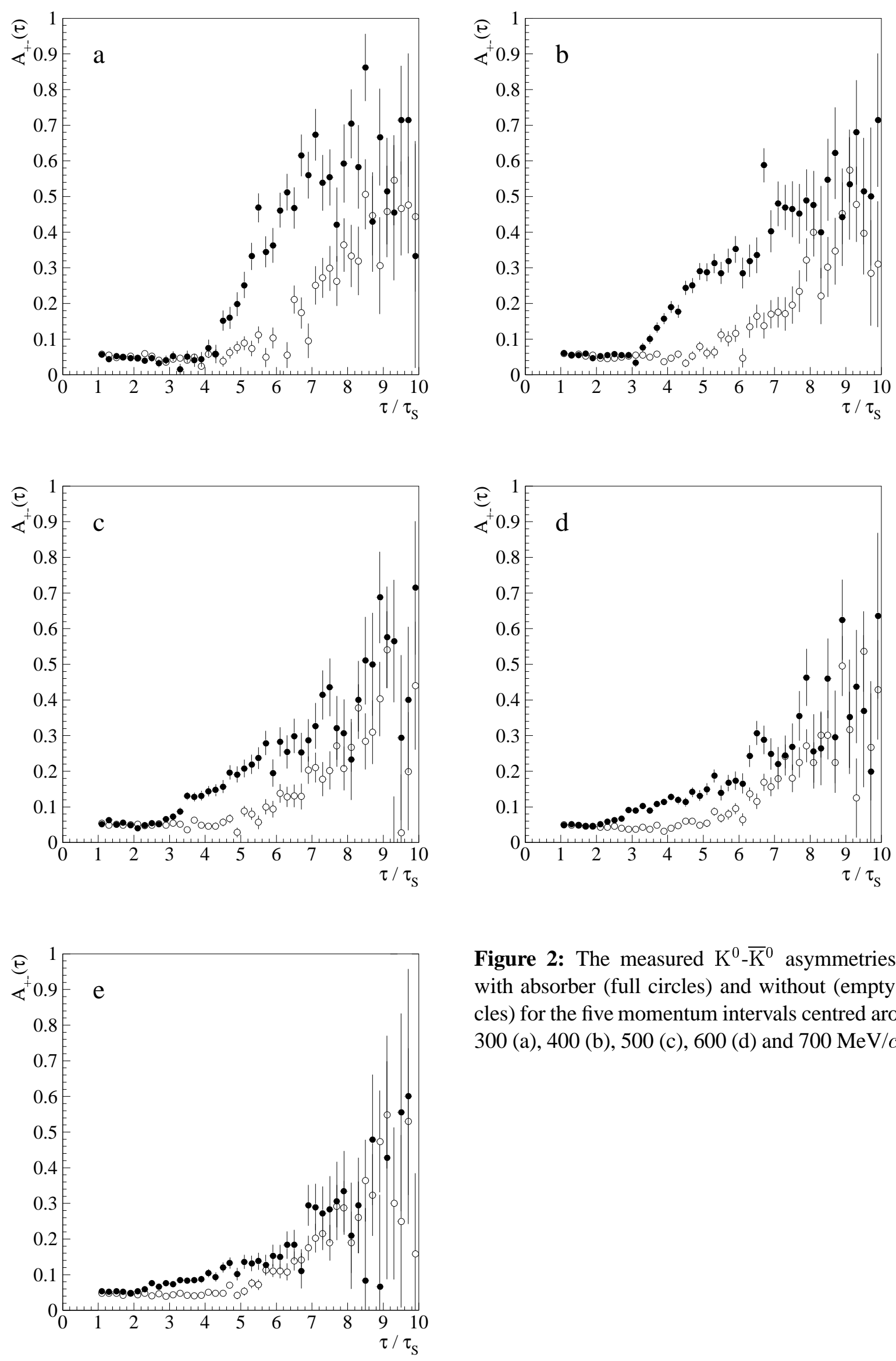

Figure 2: The measured $\mathrm{K}^{0}-\overline{\mathrm{K}}^{0}$ asymmetries (1) with absorber (full circles) and without (empty circles) for the five momentum intervals centred around 300 (a), 400 (b), 500 (c), 600 (d) and $700 \mathrm{MeV} / \mathrm{c}$ (e). 


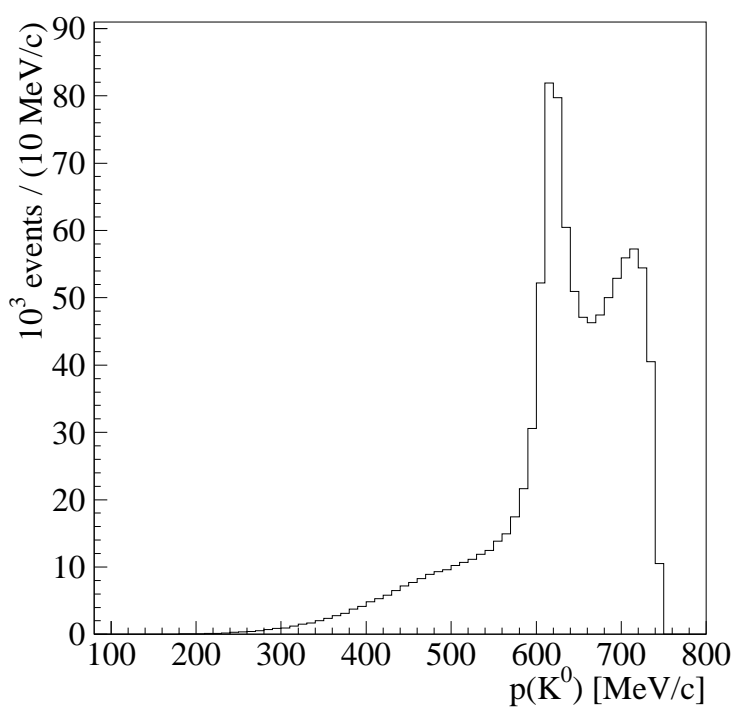

Figure 3: The $\mathrm{K}^{0}$ momentum spectrum at the entrance of the absorber. The peak at $614 \mathrm{MeV} / c$ arises from $\mathrm{K}^{0}$ s recoiling against a $\mathrm{K}^{*}(892)^{0}$ resonance.

\section{Systematic uncertainties}

In our experiment, the tagging efficiency for $\overline{\mathrm{K}}^{0}$ is higher than for $\mathrm{K}^{0}$ because the strong interaction of charged kaons and pions with the detector material favour the detection of the accompanying $\left(\mathrm{K}^{+} \pi^{-}\right)$pair over its charge conjugate. This leads to an offset (normalization factor) in the measured asymmetry which is clearly visible in Fig. 2. The factor is expected to be of the same magnitude in both the regeneration and the reference data set, i.e. the relative normalization factor $\alpha_{\text {rel }}$ should be close to one. We measured this factor by comparing the observed ratios of $\overline{\mathrm{K}}^{0}$ and $\mathrm{K}^{0}$ decays before the absorber in both data sets and obtained $\alpha_{\text {rel }}=$ $1.0028 \pm 0.0016$, constant in phase space. In the analysis, we applied this factor as a correction to the reference data set and deduced the systematic errors by varying it in its range of statistical uncertainty. This resulted in the errors given in Table 1.

Table 1: Systematic errors on $\Delta f$ due to the uncertainty in the normalization of $\mathrm{K}^{0}$ and $\overline{\mathrm{K}}^{0}$ rates.

\begin{tabular}{ccc}
\hline $\begin{array}{c}\text { mom. range } \\
\mathrm{MeV} / c\end{array}$ & $\begin{array}{c}\operatorname{Re}(\Delta f) \\
\mathrm{fm}\end{array}$ & $\begin{array}{c}\operatorname{Im}(\Delta f) \\
\mathrm{fm}\end{array}$ \\
\hline & & \\
$250-550$ & $<0.05$ & $<0.05$ \\
$550-650$ & 0.06 & 0.2 \\
$650-750$ & 0.06 & 0.6 \\
\hline
\end{tabular}

A further systematic error is caused by the uncertainty of $\pm 0.5 \mathrm{~mm}$ in the distance between the absorber and the $\mathrm{K}^{0}\left(\overline{\mathrm{K}}^{0}\right)$ production point. This uncertainty propagates to differences in the regeneration amplitudes that do not exceed $0.05 \mathrm{fm}$ in both the real and imaginary parts of the amplitudes.

Any difference in running conditions between the collection of the two data samples used for the analysis could constitute a further source of systematic error. In addition to ensuring that 
all detector and trigger parameters remained the same, we verified that neither the presence of the absorber nor the time difference of one year between the two runs affected the data in any unpredictable way. This was done by analyzing events in which no particle came close to the absorber. The asymmetries constructed from these events are equal for the two data sets within statistical fluctuations.

We also carefully studied the effect of incoherent (diffractive) regeneration which is not taken into account in our analysis. It can be shown that in $\mathrm{K}^{0}-\overline{\mathrm{K}}^{0}$ asymmetry experiments the influence of incoherent regeneration is very small for measurements in the $K_{S}$-dominated decay region and may be neglected in our analysis.

Furthermore we looked at the systematics already considered for the $\eta_{+-}$precision measurement [7], namely background level, decay time resolution, regeneration in other detector components and the uncertainties in $\Delta m$ and $\tau_{\mathrm{S}}$. These effects were studied with essentially the same tools as described in Ref. [7] and all found to be negligible in this analysis.

\section{$7 \quad$ Additional constraints on $\operatorname{Im}(\Delta f)$ from other experiments}

The optical theorem relates total cross sections to the imaginary parts of the corresponding forward scattering amplitudes. Under the assumption of isospin invariance in kaon nucleon scattering, measurements of the difference between $\mathrm{K}^{+}$and $\mathrm{K}^{-}$total cross sections may therefore be employed to put further constraints on our results and possibly resolve their large correlation present in some momentum bins.

Unfortunately we know of only one such measurement that overlaps with our momentum range. Bugg et al. [11] have measured $\mathrm{K}^{+}$and $\mathrm{K}^{-}$total cross sections in carbon at momenta between $655 \mathrm{MeV} / c$ and $2606 \mathrm{MeV} / c$, also motivated by the study of regeneration. (Krauss et al. [12] obtained data at lower momenta but only for $\mathrm{K}^{+}$.)

We interpolated the values of Ref. [11] to compare them with our measurement in the momentum range $650-750 \mathrm{MeV} / \mathrm{c}$ and found excellent agreement. The combination of the two measurements reduces the error on the real part of $\Delta f$ by more than a factor of 6 (see Fig. $4 \mathrm{e}$ and Table 2).

Table 2: The CPLEAR results on $\Delta f$ with standard errors in the Gaussian approximation in comparison with the optical model calculations of Ref. [3] where available; in the last row the combined result of Ref. [11] and CPLEAR. The values of Refs. [3] and [11] are weighted according to the momentum spectrum of Fig. 3 .

\begin{tabular}{cccccc}
\hline \multicolumn{3}{c}{ CPLEAR } & \multicolumn{2}{c}{ optical model [3] } \\
\hline$p_{\mathrm{K}^{0}}$ & $\langle\operatorname{Re}(\Delta f)\rangle$ & $\langle\operatorname{Im}(\Delta f)\rangle$ & correl. & $\langle\operatorname{Re}(\Delta f)\rangle$ & $\langle\operatorname{Im}(\Delta f)\rangle$ \\
$\mathrm{MeV} / c$ & $\mathrm{fm}$ & $\mathrm{fm}$ & coeff. & $\mathrm{fm}$ & $\mathrm{fm}$ \\
\hline & & & & & \\
$250-350$ & $-3.8 \pm 1.3$ & $-2.4 \pm 1.2$ & -0.76 & & \\
$350-450$ & $-4.9 \pm 0.5$ & $-1.2 \pm 1.6$ & -0.76 & -3.48 & -3.88 \\
$450-550$ & $-4.2 \pm 0.3$ & $-4.9 \pm 2.0$ & 0.60 & -4.03 & -3.38 \\
$550-650$ & $-5.1 \pm 0.6$ & $-2.8 \pm 1.9$ & 0.96 & -5.26 & -3.81 \\
$650-750$ & $-5.7 \pm 1.8$ & $-4.3 \pm 3.6$ & 0.99 & -6.38 & -4.59 \\
& & & & & \\
$650-750$ & $-5.5 \pm 0.3$ & $-4.1 \pm 0.2$ & & & \\
\hline
\end{tabular}



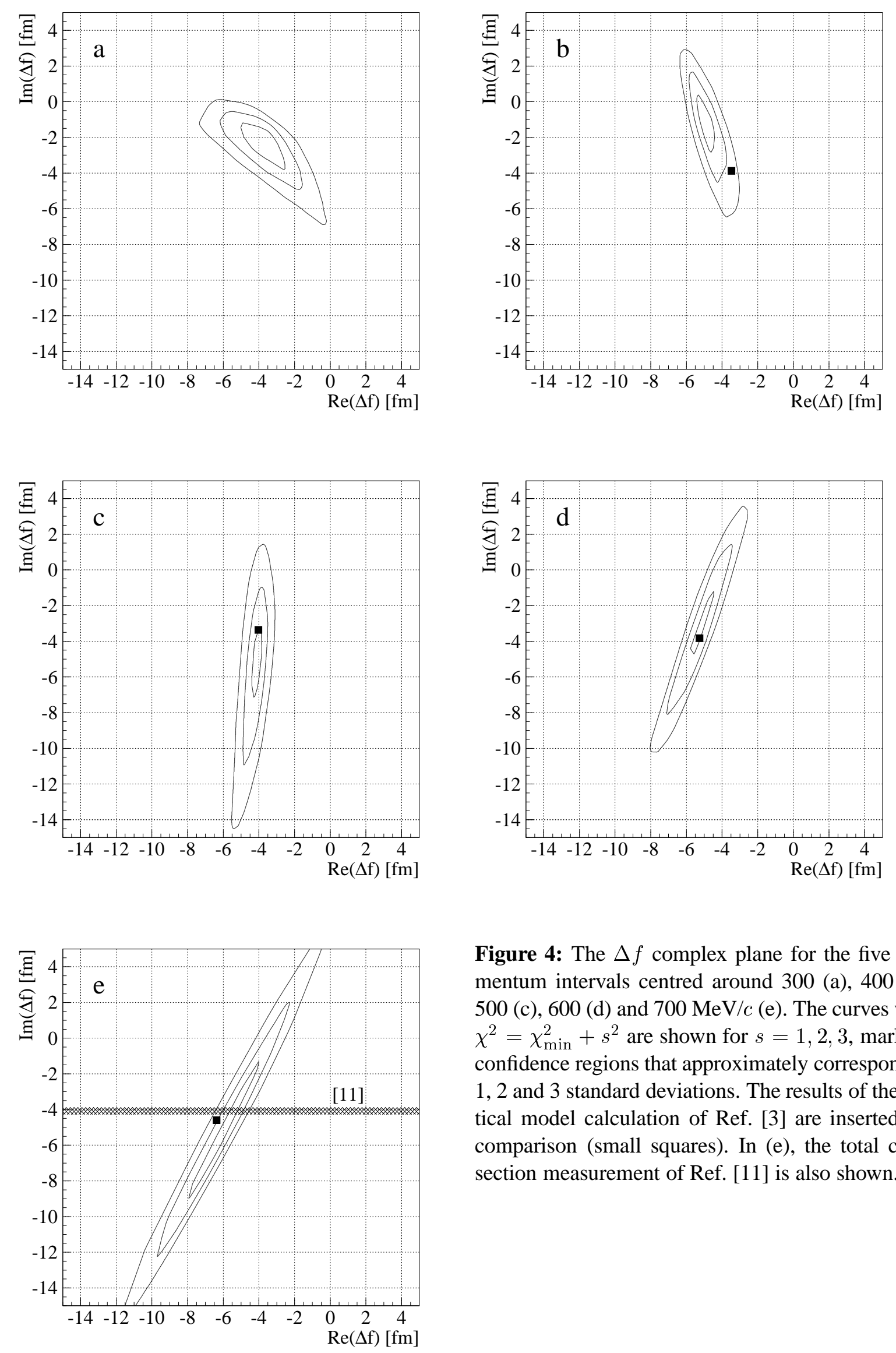

Figure 4: The $\Delta f$ complex plane for the five momentum intervals centred around 300 (a), 400 (b), 500 (c), 600 (d) and $700 \mathrm{MeV} / \mathrm{c}$ (e). The curves with $\chi^{2}=\chi_{\text {min }}^{2}+s^{2}$ are shown for $s=1,2,3$, marking confidence regions that approximately correspond to 1, 2 and 3 standard deviations. The results of the optical model calculation of Ref. [3] are inserted for comparison (small squares). In (e), the total cross section measurement of Ref. [11] is also shown. 


\section{Final results and conclusion}

Our results are summarized in Fig. 4 and Table 2. They represent the first experimental data on both the real and imaginary parts of the regeneration amplitude for kaon momenta below $1 \mathrm{GeV} / c$ and are in accordance with the predictions of Eberhard and Uchiyama [3] as well as with more recent calculations done for the $\phi$-factory at Frascati [13].

These results on $\Delta f$ allow us to reduce the systematic error on our measurement [9] of the $\mathrm{CP}$ violation parameter $\phi_{+-}$due to regeneration to below $0.2^{\circ}$, less than half of the statistical error.

\section{Acknowledgements}

We would like to thank the CERN LEAR staff for their support and co-operation as well as the technical and engineering staff of our institutes. We are also grateful to the staff of the Institute of Biomedical Engineering at ETH Zürich for performing the tomography of the absorber. This work was supported by the following institutions: the French CNRS/Institut de Physique Nucléaire et de Physique des Particules, the French Commissariat à l'Energie Atomique, the Greek General Secreteriat of Research and Technology, the Netherlands Foundation for Fundamental Research on Matter (FOM), the Portuguese JNICT, the Ministry of Science and Technology of the Republic of Slovenia, the Swedish Natural Science Research Council, the Swiss National Science Foundation, the UK Particle Physics and Astronomy Research Council (PPARC), and the US National Science Foundation.

\section{References}

[1] M.L. Good, Phys. Rev. 106 (1957) 591.

[2] K. Kleinknecht, Fortschritte der Physik 21 (1973) 57.

[3] P.H. Eberhard, F. Uchiyama, Nucl. Instr. and Meth. A 350 (1994) 144.

[4] For higher energy data on regeneration in carbon see K.-F. Albrecht et al., Nucl. Phys. B 93 (1975) 237;

W.C. Carithers et al., Phys. Rev. Lett. 34 (1975) 1240;

J. Roehrig et al., Phys. Rev. Lett. 38 (1977) 1116.

[5] E. Gabathuler and P. Pavlopoulos, Strong and Weak CP violation at LEAR, in: Proc. Workshop on Physics at LEAR with Low Energy Cooled Antiprotons (Erice, 1982), eds. U. Gastaldi and R. Klapisch (Plenum, New York, 1984) p. 747.

[6] CPLEAR Collab., R. Adler et al., Nucl. Instr. and Meth. A 379 (1996) 76.

[7] CPLEAR Collab., R. Adler et al., Phys. Lett. B 363 (1995) 243.

[8] W. Fetscher et al., Z. Phys. C 72 (1996) 543.

[9] CPLEAR Collab., A determination of the $\mathrm{CP}$ violation parameter $\eta_{+-}$from the decay of strangeness-tagged neutral kaons, to be published.

[10] Particle Data Group, R.M. Barnett et al., Phys. Rev. D 54 (1996) 1.

[11] D.V. Bugg et al., Phys. Rev. 168 (1968) 1466.

[12] R.A. Krauss et al., Phys. Rev. C 46 (1992) 655;

see also R. Weiss et al., Phys. Rev. C 49 (1994) 2569.

[13] R. Baldini, A. Michetti, LNF-96/008(IR) (1996), unpublished. 